\title{
Histone Methyltransferases as Therapeutic Targets for Kidney Diseases
}

\author{
Chao $\mathrm{Yu}^{1}$ and Shougang Zhuang ${ }^{1,2 *}$ \\ ${ }^{1}$ Department of Nephrology, Shanghai East Hospital, Tongji University School of Medicine, Shanghai, China, ${ }^{2}$ Department of \\ Medicine, Rhode Island Hospital and Alpert Medical School, Brown University, Providence, RI, United States
}

OPEN ACCESS

Edited by:

Utpal Sen,

University of Louisville, United States

Reviewed by: Xiaoxin Wang, Georgetown University Medical Center, United States

Rohan Samarakoon, Albany Medical College, United States

*Correspondence:

Shougang Zhuang szhuang@lifespan.org

Specialty section:

This article was submitted to

Renal Pharmacology,

a section of the journal

Frontiers in Pharmacology

Received: 06 May 2019

Accepted: 31 October 2019

Published: 06 December 2019

Citation:

Yu C and Zhuang S (2019) Histone Methyltransferases as Therapeutic

Targets for Kidney Diseases.

Front. Pharmacol. 10:1393.

doi: 10.3389/fphar.2019.01393
Emerging evidence has demonstrated that epigenetic regulation plays a vital role in gene expression under normal and pathological conditions. Alterations in the expression and activation of histone methyltransferases (HMTs) have been reported in preclinical models of multiple kidney diseases, including acute kidney injury, chronic kidney disease, diabetic nephropathy, polycystic kidney disease, and renal cell carcinoma. Pharmacological inhibition of these enzymes has shown promise in preclinical models of those renal diseases. In this review, we summarize recent knowledge regarding expression and activation of various HMTs and their functional roles in some kidney diseases. The preclinical activity of currently available HMT inhibitors and the mechanisms of their actions are highlighted.

Keywords: chronic kidney diseases, epigenetic regulations, histone modification, histone methyltransferases, expression

\section{INTRODUCTION}

Epigenetics is a heritable change in gene expression, including covalent modifications of DNA and related histone proteins in the absence of an altered change in DNA sequence. In general, epigenetic regulation involving histone modification, DNA methylation, and non-coding RNAs can alter the phenotype but not the genotype in gene expression (Peschansky and Wahlestedt, 2014; Reddy and Natarajan, 2015). Histone modification involves distinct types of post-translational modifications (PTMs) of core histone proteins, including acetylation, methylation, phosphorylation, and ubiquitination (Susztak, 2014). These modifications occur primarily on the amino terminal ends of the histones within the nucleosome core. They are thought to change the structure and transcriptional process of chromatin, thereby influencing the downstream process of gene expression positively or negatively, alone or in combination with other modifications of gene expression (Chen et al., 2014b).

Histone methylation commonly occurs in specific lysine and arginine residues at the amino terminal ends of histones core (Greer and Shi, 2012). Each lysine has three possible methylation states: mono-methylated, di-methylated or tri-methylated with methyl groups covalently bonded to the lysine $\varepsilon$-amine group. In contrast, arginine post-methylation can exist in mono-methylated, di-methylated symmetrical and asymmetrical forms with methylation of the molecule's guanidyl group (Bedford and Richard, 2005). Differences in residue methylation and modification states correlate with whether histone methylation activates or represses transcription (Li et al., 2007). For example, lysine methylation at H3 lysine 4 (H3K4), H3K36, and H3K79 is associated with transcriptional activation. Conversely, methylation at $\mathrm{H} 3 \mathrm{~K} 9, \mathrm{H} 3 \mathrm{~K} 27$, and $\mathrm{H} 4 \mathrm{~K} 20$ is related to gene repression (Sims et al., 2003). The underlying mechanism by which the active and repressive actions can operate in such a coordinated manner remains incompletely understood. 
Histone methyltransferases (HMTs) are a class of enzymes that mediate the methylation of lysine or arginine residues of histones. So far, more than 50 lysine human methyltransferases (KMTs) have been reported. These enzymes have high selectivity for the histone lysine residue they target and are classified into two types: lysine methyltransferases (KMTs) and arginine methyltransferases (PRMTs). Based on catalytic domain sequence, KMTs are further divided into two families: SET domain-containing KMTs, which include Su(var)3-9, Enhancer of Zeste (EZH), Trithorax, and non-SET domain-containing KMTs, such as the DOT1-like proteins (Feng et al., 2002; Ng et al., 2002; Herz et al., 2013). The structure of SET methyltransferase contains a SET domain, a pre-SET, and a post-SET domain. SET methyltransferases are further sub-divided into different families. The SET1 family bears the SET domain usually followed by a post-SET domain. Two well-known methyltransferases, EZH1, and EZH2, belong to this family although they do not have the post-SET domain. The SET domain in the SET2 family is always flanked by a post-SET and an AWS domain, where the nuclear receptor binds to the SET domain, which contains proteins such as NSD1-3, the SETD2 and the SMYD family proteins. Members of the SUV39 family all demonstrate a pre-SET domain that includes SUV39H1, SUV39H2, G9a, GLP, ESET, and CLLL8 (Rea et al., 2000). Still other SET domain-containing methyltransferases have not been categorized into specific sub-groups, like SET7/9, SET8, SUV4-20H1, and SUV4-20H2 (Dillon et al., 2005). The human DOT1-like (DOT1L) protein is a non-SET domain containing methyltransferase that methylates a lysine residue in the globular core of the histone (Wood and Shilatifard, 2004). Among the PRMTs, there are three types of methylation patterns based on different arginine binding pockets. The first type of PRMTs, including PRMT1, PRMT3, and cofactor associated arginine methyltransferase (CARM1), can produce monomethyl arginine and asymmetric dimethylarginine (Chen et al., 1999; McBride et al., 2000). The second type of PRMTs, including PRMT5, can produce monomethyl or symmetric dimethylarginine (Branscombe et al., 2001). The third type of PRMTs, including PRMT7, only produces monomethylated arginine (Blanc and Richard, 2017) (Table 1).

Histone methylation plays important roles in many biological processes, including cell cycle regulation, stress response, development, and differentiation. Initial studies indicated that $\mathrm{H} 3$ methylation on lysines 4, 27, and 79 plays an essential part in regulation of gene expression in the developing kidney (McLaughlin et al., 2014). Recent studies have demonstrated that abnormal expression or activity of histones methyltransferases are associated with development and prognostic state of various kidney diseases in human and animal models such as acute kidney injury (AKI), chronic kidney disease (CKD), diabetic nephropathy (DN), polycystic kidney disease, and renal cell carcinoma (Portela and Esteller, 2010; Takawa et al., 2011; Dressler and Patel, 2015; McGrath and Trojer, 2015; Cai et al., 2016; Siddiqi et al., 2016). This review focuses on recent reports on the expression and role of histone methyltransferases in various kidney diseases and their potential as targets for the treatment of these diseases.

\section{EZH2}

$\mathrm{EZH} 2$, the functional enzymatic component of polycomb repressor complex 2 (PRC2), is responsible for the trimethylation of histone $\mathrm{H} 3$ lysine 27 (H3K27me3). EZH2 and the whole PRC2 are critical for regulating a number of genes involved in development and tumorigenesis. In the kidney, EZH2 transcripts are detectable

TABLE 1 | Classification of histone methyltransferases.

\begin{tabular}{|c|c|c|c|c|}
\hline Methyltransferase & Type & & Site & Function \\
\hline \multirow[t]{19}{*}{ KMTs } & SET1 family & $\mathrm{EZH} 1$ & H3K27 & Transcriptional repression \\
\hline & SET1 family & H3K27 & $\mathrm{H} 2 \mathrm{~K} 27$ & Transcriptional repression \\
\hline & SET2 family & NSD1-3 & H3К36 & Transcriptional activation \\
\hline & SET2 family & SETD2 & H3К36 & Transcriptional activation \\
\hline & SET2 family & SMYD2 & H3K36/p53 & Transcriptional activation \\
\hline & SUV39 family & SUV39H1 & H3K9 & Heterochromatin formation/silencing \\
\hline & SUV39 family & SUV39H2 & $\mathrm{H} 3 \mathrm{~K} 9$ & Heterochromatin formation/silencing \\
\hline & SUV39 family & G9a & H3K9 & Heterochromatin formation/silencing \\
\hline & SUV39 family & GLP & $\mathrm{H} 3 \mathrm{~K} 9$ & Heterochromatin formation/silencing \\
\hline & SUV39 family & ESET//SETDB1 & H3K9 & Transcriptional repression \\
\hline & SUV39 family & CLLL8/SETDB2 & & \\
\hline & RIZ family & $\mathrm{RIZ1}$ & H3K9 & Transcriptional repression \\
\hline & RIZ family & BLIMP1/PRDM1 & & \\
\hline & RIZ family & PFM1/CRS2 & & \\
\hline & Non-group family & SET7/9 & H3K4/p53/TAF10 & Transcriptional repression \\
\hline & Non-group family & SET8 & $\mathrm{H} 4 \mathrm{~K} 20$ & Transcriptional repression \\
\hline & Non-group family & SUV4-2OH1 & $\mathrm{H} 4 \mathrm{~K} 20$ & DNA damage response \\
\hline & Non-group family & SUV4-2OH2 & $\mathrm{H} 4 \mathrm{~K} 20$ & \\
\hline & non-SET family & DOT1L & H3К79 & Transcriptional activation \\
\hline \multirow[t]{5}{*}{ PRMTs } & I & PRMT1 & H4R3 & Transcriptional activation \\
\hline & 1 & PRMT3 & RPS2 & Transcriptional activation \\
\hline & 1 & PRMT4 /CARM1 & H3R2, H3R17, H3R26 & Transcriptional activation \\
\hline & $\|$ & PRMT5 & H3R8, H4R3 & Transcriptional repression \\
\hline & III & PRMT7 & H4R3 & Imprinting in male germ cell \\
\hline
\end{tabular}


throughout embryonic development (Caretti et al., 2004), but absent in adult kidneys. During renal development, a moderate level of EZH2 protein is detectable in the nuclei of renal tubular epithelial cells at E14.5, but not in glomeruli; in fetal kidneys at E18.5, a small amount of EZH2 protein is also observed in the cytoplasm of renal tubular epithelial cells (Ningxia et al., 2011). The localization of EZH2 protein within cells at different stage of development suggests its distinct regulatory role at early and later periods of organogenesis. EZH2 has been reported to be required for both cellular proliferation and differentiation in the process of organogenesis (Martinez and Cavalli, 2006; Oktaba et al., 2008).

Increasing evidence indicates that the abnormal expression or activity of EZH2 is associated with the development of chronic renal disease. In a murine model of renal fibrosis induced by unilateral ureteral obstruction, EZH2 is expressed in myofibroblasts and injured renal tubular cells. Similarly, increased EZH2 levels have been detected in those two cell types in kidney biopsy samples of patients with focal segmental glomerulosclerosis and IgA nephropathy, but not with minimal change disease (Zhou et al., 2016) (Table 3). Mechanistic studies showed that downregulation of EZH2 by inhibitors or siRNA reduces the activation of renal interstitial fibroblasts and epithelial-to-mesenchymal transition (EMT) of renal tubular cells in vitro; treatment with the EZH2 inhibitor 3-deazaneplanocin A (3-DZNeP) also attenuates UUOinduced renal fibroblast activation, partial EMT and fibrosis in mice (Zhou et al., 2016; Zhou et al., 2018a). The antifibrotic actions of EZH2 blockade are associated with dephosphorylation of multiple profibrotic receptors, including epidermal growth factor receptor and platelet derived growth factor receptor and inactivation of several intracellular signaling pathways, including Smad3, signal transducer and activator of transcription 3 (STAT3), extracellular signal-regulated kinase 1/2 (ERK1/2) and AKT (Vignais et al., 1996; Wang et al., 2005; Zhou et al., 2016). Moreover, we found that EZH2 expression is required for renal epithelial cells arrested at the G2/M phase of the cell cycle (Zhou et al., 2016; Zhou et al., 2018a) to change into a cell phenotype that is able to produce and release an excessive amount of growth factors and cytokines, thereby inducing the transition of fibroblasts into myofibroblasts (Grande et al., 2015; Lovisa et al., 2015). As development of partial EMT in renal epithelial cells is driven by Snaill and Twist, two transcriptional factors, we also examined the role of EZH2 in the regulation of their expression following UUO injury. We showed that blocking EZH2 with 3-DZNeP led to decreased expression of Snail1 and Twist (Zhou et al., 2018a). Collectively, these data suggest that EZH2 is critically involved in the regulation of renal fibroblast activation, partial EMT and renal fibrosis. In contrast to our observations, it appears that EZH2 protects against podocyte oxidative stress and renal injury in diabetes. This is evidenced by data showing that pharmacologic or genetic depletion of EZH2 induced podocyte injury and augmented oxidative stress and proteinuria in diabetic mice (Siddiqi et al., 2016) (Table 2). Furthermore, genetic depletion and pharmacological inhibition of EZH2 promotes podocyte injury and glomerular in murine models of nephrotoxicity and subtotal nephrectomy (Majumder et al.,

TABLE 2 | Histone methyltransferase inhibition in kidney diseases.

\begin{tabular}{|c|c|c|c|c|c|}
\hline KMTs & Histone targets & $\begin{array}{l}\text { Inhibitors or } \\
\text { Knockout mice }\end{array}$ & Model & Effects & References \\
\hline \multirow[t]{7}{*}{$\mathrm{EZH} 2$} & H3K27 & 3-DZNeP & UUO & $\begin{array}{l}\text { Inhibits renal fibroblast activation and EMT, alleviates } \\
\text { renal fibrosis }\end{array}$ & $\begin{array}{l}\text { (Zhou et al., 2016; } \\
\text { Zhou et al., 2018a) }\end{array}$ \\
\hline & & 3-DZNeP & I/R or FA induced $\mathrm{AKI}$ & Alleviates AKI and reduces renal tubular cell death & (Zhou et al., 2018b) \\
\hline & & 3-DZNeP & Cisplatin induced AKI & Protects against renal tubular cell death, inhibits AKI & (Ni et al., 2019) \\
\hline & & 3-DZNeP & Hyperuricemia-induced CKD & $\begin{array}{l}\text { Inhibits fibroblast activation and proliferation, attenuates } \\
\text { renal injury }\end{array}$ & (Shi et al., 2019) \\
\hline & & $\begin{array}{l}\text { 3-DZNeP } \\
\text { KO mice } \\
\text { EPZ-6438, KO mice }\end{array}$ & $\begin{array}{l}\text { STZ-induced } \\
\text { DN, Adriamycin } \\
\text { nephrotoxicity, SNx }\end{array}$ & $\begin{array}{l}\text { Induces podocyte injury, increases oxidative stress and } \\
\text { proteinuira Sensitize mice to glomerular disease increase } \\
\text { podocyte injury and dedifferentiation }\end{array}$ & (Siddiqi et al., 2016) \\
\hline & & & Same models as above & $\begin{array}{l}\text { Sensitize mice to glomerular disease increase podocyte } \\
\text { injury and dedifferentiation }\end{array}$ & $\begin{array}{l}\text { (Majumder et al., } \\
\text { 2018) }\end{array}$ \\
\hline & & GSK126 & $\begin{array}{l}\text { Rat renal interstitial fibroblast } \\
\text { cells }\end{array}$ & Inhibits activation of cultured renal interstitial fibroblasts & (Zhou et al., 2016) \\
\hline G9a & $\mathrm{H} 3 \mathrm{~K} 9$ & $\mathrm{BIX01294}$ & UUO & Attenuates renal fibrosis and retains klotho expression & (Irifuku et al., 2016) \\
\hline SUV39H1 & H3K9 & Chaetocin & Mesangial cells & Increases fibronectin and p21expression & (Lin et al., 2016) \\
\hline SET7/9 & $\mathrm{H} 3 \mathrm{~K} 4$ & Sinefungin & UUO & Ameliorates renal fibrosis; Suppresses peritoneal fibrosis & (Sasaki et al., 2016) \\
\hline DOT1L & H3К79 & EPZ5676 & UUO & $\begin{array}{l}\text { Inhibits renal fibroblast activation and EMT; attenuates } \\
\text { renal fibrosis }\end{array}$ & (Liu et al., 2019) \\
\hline SMYD2 & $\mathrm{H} 3 \mathrm{~K} 4 ; \mathrm{H} 3 \mathrm{~K} 36$ & AZ505 KO mice & Hypomorphic Pkd1nl/nl mice & $\begin{array}{l}\text { Delay cyst growth in ADPKD, reduce activation of STAT3 } \\
\text { and NF-kB }\end{array}$ & (Li et al., 2017) \\
\hline
\end{tabular}

DN, Diabetic nephropathy; STZ, streptozotocin; CKD, chronic kidney disease; UUO, unilateral ureteral obstruction; SNx, subtotal nephrectomy; KO, knockout mice; AKI, acute kidney injury; EMT; epithelial-mesenchymal transition; ADPKD, autosomal dominant polycystic kidney disease; STAT3, signal transducer and activator of transcription 3; NF- $\kappa B$, nuclear factor kappa light chain enhancer of activated $B$ cells. 
2018) (Table 2). The distinct functions of EZH2 in renal tubules and podocytes are not clear, but may reflect differences in location and targets in the kidney. In podocytes, EZH2 upregulation leads to repression of endogenous antioxidant inhibitor thioredoxin interacting protein (TxnIP), a molecule that inhibits the actions of the endogenous antioxidant thioredoxin and promotes oxidative injury (Siddiqi et al., 2016).

EZH2 also contributes to the pathogenesis of acute kidney injury (AKI). In mouse models of ischemia/reperfusion or folic acid-induced AKI, EZH2 and H3K27 are highly expressed in the injured tubules. Pharmacological inhibition of EZH2 lessens pathological changes and attenuates renal tubule injury and cell death; inhibition of EZH2 with either 3-DZNep or its specific siRNA also promotes survival of renal epithelial cells in culture following oxidant injury (Zhou et al., 2018b). Moreover, EZH2 inhibition increases expression of its multiple target molecules, including E-cadherin, TIMP-2/3, and RKIP, which are associated with renal tubular injury (Zhou et al., 2018b). In line with our observations, Liang et al. (2019) demonstrated that treatment with 3-DZNep improves renal dysfunction, lessens tubular injury, reduces production of several cytokines, including TNFa, MCP-1 IL-6 and IL-18 and decreases activation of p38, a stress protein in mice after ischemia/reperfusion. Furthermore, Li et al. (2016a) reported that inhibition of EZH2 with 3-DZNep resulted in amelioration of early acute renal allograft rejection in rats through suppression of activation of alloreactive $\mathrm{T}$ cells and subsequent production of multiple inflammatory cytokines. Thus, EZH2 is critically involved in the acute inflammatory response and renal injury. Recently, we further demonstrated that administration of 3-DZNep was protective in a murine model of AKI induced by cisplatin through a mechanism involved in the preservation of E-cadherin (Ni et al., 2019).

Collectively, these studies indicate that EZH2 plays a detrimental role in renal tubules but a protective role in podocytes. Therefore, targeting EZH2 may be a potential approach for the treatment of renal fibrosis, AKI and acute rejection after transplantation, but not for diabetic nephropathy. Given that EZH2 plays a protective role in podocytes, EZH2 inhibitors should be avoided in renal diseases characterized by podocyte injury.

\section{G9a}

Among the histone modification enzymes, G9a is a key histone lysine methyltransferase that can catalyze mono- and dimethylation at $\mathrm{H} 3 \mathrm{~K} 9$ (mel and me2), a repressive chromatin marker (Tachibana et al., 2001; Tachibana et al., 2002). In the developing kidney, G9a is mainly expressed in the Pax $2^{+}$cap mesenchyme and the distal and proximal portions of nascent nephrons (McLaughlin et al., 2014). Several studies have revealed that G9a is upregulated in various human tumors, including colorectal cancer, breast cancer, ovarian cancer, and bladder cancer (Huang et al., 2010; Hua et al., 2014; Zhang et al., 2015; Chen et al., 2017; Cao et al., 2019) and plays a role in tumorigenesis. G9a activity can also be induced by transforming growth factor- $\beta 1$ (TGF- $\beta 1$ ) and is involved in the development of epithelial mesenchymal transposition in cancer cells (Dong et al., 2012). Given that TGF- $\beta 1$ is a pivotal cytokine in tissue fibrosis, Irifuku et al. (2016) examined the expression of G9a and its role in a murine model of UUO-induced renal fibrosis by administration of G9a small interfering RNA and BIX01294, a specific inhibitor of G9a. They found that G9a expression levels were upregulated in the murine kidney of UUO and in human kidney biopsy specimens obtained from patients with IgA nephropathy (Table 3). Treatments with interfering RNA and BIX01294 reduced activation of renal interstitial fibroblasts and accumulation of ECM proteins, which is accompanied by increased klotho expression and decreased methylation of $\mathrm{H} 3 \mathrm{~K} 9$. In vitro, they also observed that treatment with BIX01294 inhibited TGF- $\beta 1$-induced fibrotic changes and klotho downregulation in renal epithelial cells (Irifuku et al., 2016). In a separate study, the same group found that pharmacological inhibition of G9a attenuated peritoneal fibrosis and improved peritoneal function in a murine model of peritoneal fibrosis induced by methylglyoxal (Maeda et al., 2017). Nevertheless, the regulatory role of G9a is not limited to renal and peritoneal fibrosis. G9a has also been reported to play a role in the pathogenesis of idiopathic pulmonary fibrosis (Coward et al., 2018). Thus, G9a may mediate tissue fibrosis in different organs, but there are so far no reports about its role in acute organ injury. One group, however, reported that BIX01294 treatment protects hair cells from aminoglycoside-induced damage in mice (Yu et al., 2013), suggesting that like EZH2, G9a may also be involved in the pathogenesis of AKI. Further investigation into this issue is needed.

\section{SUV39H1}

The histone methyltransferase SUV39H1 catalyzes the trimethylation of histone $\mathrm{H} 3$ lysine 9 (H3K9me3) and is a wellknown transcriptional repressor of inflammatory genes. The impact of SUV39H1 on inflammatory gene promoters under the stimulation of high-glucose has been reported in various cell lines, including vascular smooth muscle cells (VSMCs) (Villeneuve

TABLE 3 | Expression of HMTs in human kidney diseases.

\begin{tabular}{|c|c|c|c|c|}
\hline Human kidney disease & HMTs & & Histone Targets & References \\
\hline Focal segmental glomerulosclerosis, & $\mathrm{EZH} 2$ & upregulated & H3K27 & (Zhou et al., 2016; Zhou et al., 2018a) \\
\hline IgA nephropathy & $\mathrm{EZH} 2$ & upregulated & H3К27 me3 & \\
\hline IgA nephropathy & G9a & upregulated & H3K9 & (Irifuku et al., 2016) \\
\hline Diabetic nephropathy & SUV39H1 & upregulated & H3K7 & (Wang et al., 2018b) \\
\hline IgA and membranous nephropathy & SET7/9 & upregulated & $\mathrm{H} 3 \mathrm{~K} 4$ & (Sasaki et al., 2016) \\
\hline Polycystic kidneys & SMYD2 & upregulated & H3К4, Н3К36 & (Pires-Luis et al., 2015; Li et al., 2017) \\
\hline Membranous nephropathy & MLL3 & Upregulated & H3K4 H3К36 & (Fujino and Hasebe, 2016) \\
\hline Focal segmental glomerulosclerosis & $\mathrm{EZH} 2$ & upregulated & H3К27 & (Wang et al., 2019) \\
\hline
\end{tabular}


et al., 2008), macrophages (Li et al., 2016b), and cardiomyocytes (Yang et al., 2017b). Recent studies suggest that SUV39H1 and $\mathrm{H} 3 \mathrm{~K} 9 \mathrm{me} 3$ were overexpressed in renal tubules of patients with diabetic nephropathy (Wang et al., 2018b) (Table 3), indicating that their expression correlates with the development of chronic kidney disease. However, hyperglycemia decreased expression of SUV39H1 in type 1 diabetes-associated renal fibrosis (Goru et al., 2016). Further studies showed that high glucose-induced downregulation of $\mathrm{H} 3 \mathrm{~K} 9 \mathrm{me} 3$ and SUV39H1 primarily occurred in diabetic mouse vascular smooth muscle cells and mesangial cells (Lin et al., 2016). Interestingly, genetic or pharmacological inhibition of phosphoinositide 3-kinase (PI3K) reduced high glucose-induced downregulation of SUV39H1 in mesangial cells, while genetic overexpression of SUV39H1 or pharmacological increase of SUV39H1 was protective against the progression of DN (Lin et al., 2016). These findings imply that dysregulation of SUV39H1 may contribute to DN progression and that SUV39H1 may serves as a therapeutic target for the treatment of DN.

\section{Dot1/DOT1L}

The lysine methyltransferase responsible for H3K79 methylation is named by Dot1 (disruptor of telomeric silencing 1) in lower eukaryotes and Dot1-like protein (DOT1L) in mammals (Min et al., 2003). It is the only known mammalian histone methyltransferase (Min et al., 2003) that can catalyze the mono-, di-, and tri- methylation of histone $\mathrm{H} 3$ lysine 79 (H3K79) and regulate the activation or repression of gene transcription (Min et al., 2003). The mono- and di-methylation of H3K79 is associated with gene transcriptional activation, whereas H3K79 trimethylation is associated with gene repression (Wong et al., 2015). As one of the most studied histone methyltransferase, DOT1L has been shown to play a vital role in many biological processes, including mammalian development, cell cycle progression, cardiomyocyte differentiation, postembryonic development, somatic reprogramming, DNA damage response, and tumorigenesis (Huyen et al., 2004; Janzen et al., 2006; Jones et al., 2008; Wakeman et al., 2012; Wen et al., 2015; Cattaneo et al., 2016). It has been reported that increasing DOT1L expression by spironolactone or by stable transfection led to impaired endothelin-1 expression in NRK-52 cells, indicating a possible role of DOT1L in mediating the pathogenesis of diabetes (Wu et al., 2016). Further studies showed that spironolactone attenuates kidney injury by restoring DOtla, one of five splicing variants (Dotla-Dotle) of DOT1L, which mediates repression of endothelin-1 expression in streptozotocin-induced diabetic rats (Zhou et al., 2012). In addition, Dotla plays a role in the regulation of water homeostasis by targeting aquaporin 5 (Wu et al., 2013). Although the role of DOT1L in most kidney diseases is not known currently, our recent studies demonstrated that DOT1L was critically involved in the pathogenesis of renal fibrosis in a murine model of UUO-induced chronic kidney disease. DOT1L and di-methylated H3K79 were highly expressed in renal tubular epithelial cells and myofibroblasts in UUO kidneys and in in vitro cultures of these two types of cells; pharmacological inhibition of DOT1L with EPZ5676, a highly selective inhibitor of DOT1L, significantly reduced renal fibrosis (Liu et al., 2019). Treatment with EPZ5676 or DOT1L small interfering RNA also inhibited TGF- $\beta 1$ and serum-induced activation of renal interstitial fibroblasts and epithelial-mesenchymal transition (EMT) in vitro. Currently, DOT1L expression in human kidney disease remains unclear and requires further investigations.

\section{SET7/9}

SET7/9 is a protein lysine methyltransferase that methylates histone $\mathrm{H} 3 \mathrm{~K} 4$ and some nonhistone proteins such as p53 (Kurash et al., 2008). Previous studies revealed a close association between SET7/9 and neoplasms such as breast cancer, hepatocellular carcinoma, prostate cancer, and colorectal cancer (Chen et al., 2016; Huang et al., 2017; Duan et al., 2018; Wang et al., 2018a). Recently, several studies indicated that SET7/9-mediated H3K4 methylation is also related to renal disease. TGF- $\beta 1$-induced up-regulation of ECMassociated genes was accompanied by increased expression of H3K4me1/2/3 and SET7/9 (Sun et al., 2010); the level of H3K4me1 and SET7/9 recruited at MCP-1 promoters were increased in the kidneys of $\mathrm{db} / \mathrm{db}$ mice (Chen et al., 2014a); SET9 regulates TGF$\beta 1$-induced activation of renal fibroblasts through promoting Smad-3 activation (Shuttleworth et al., 2018). SET7/9 knockdown by small interfering RNAs (siRNAs) significantly inhibited MCP-1 expression and improved glomerular lesions, indicating that SET7/9-mediated H3K4me1 is involved in the expression of MCP-1 in the kidneys of db/db mice (Chen et al., 2014a). Another study showed that TGF- $\beta 1$ induced the recruitment of SET7/9 to the p21 gene promoter, while SET7/9 gene silencing with siRNAs significantly attenuated TGF- $\beta 1$-induced p 21 gene expression in rat mesangial cells (Guo et al., 2016). Further studies showed that inhibition of SET7/9 with sinefungin, a selective inhibitor of SET7/9 or siRNA attenuated renal fibrosis, as indicated by decreased expression of mesenchymal markers and ECM proteins in kidneys of UUO mice; sinefungin can also inhibit expression of TGF$\beta 1$-induced $\alpha$-smooth muscle actin expression in cultured renal epithelial cells and renal interstitial fibroblasts (Sasaki et al., 2016). Interestingly, expression of SET7/9 was also positively correlated with the degree of interstitial fibrosis in human kidneys of patients with IgA and membranous nephropathy (Sasaki et al., 2016) (Table 3). These studies provide evidence for the involvement of SET7/9 in renal fibrosis. Sasaki et al. (2016) reported that SET7/9 expression was also increased in the peritoneum in a murine model of peritoneal fibrosis induced by methylglyoxal and as well as in non-adherent cells isolated from the effluent of PD patients; treatment with sinefungin inhibited expression of collagen deposition in the peritoneum and improved peritoneal function, which is accompanied by decreased expression of $\mathrm{H} 3 \mathrm{~K} 4 \mathrm{mel}$ as well (Tamura et al., 2018).

Moreover, SET7/9 plays a role in inflammation and diabetes. Increased inflammatory gene expression and SET7/9 recruitment in macrophages were observed in diabetic mice; targeted silencing of SET7/9 with siRNA reduced TNF- $\alpha$-induced recruitment of NF- $\kappa B$ p 65 to inflammatory gene promoters (Li et al., 2008). In addition, hyperglycemia increased expression of SET7/9 in rat mesangial cells and in the kidney of type 1 diabetic rats (Goru et al., 2016). These findings suggest the possible role of SET7/9 in the development of renal fibrosis in diabetic kidney disease, but 
further investigation is needed to understand the mechanism of action of SET7/9.

Taken together, these results demonstrate that upregulation of SET7/9 and its related H3K4me1 is associated with the pathogenesis of renal and peritoneal fibrosis. Targeting SET7/9 may be a novel therapeutic treatment for fibrotic diseases.

\section{SMYD2}

SMYD2, one of the SET and MYND-containing lysine methyltransferases (SMYD), can methylate both H3K4 and H3K36 and non-histone proteins, including p53/TP53 and RB1 (Tracy et al., 2018). Increased expression of SMYD2 was initially identified in renal tumor tissue (Pires-Luis et al., 2015). Recently, Li et al. (2017) made an interesting observation that SMYD2 was also highly expressed in the polycystic renal tubular epithelial cells of autosomal dominant polycystic kidneys in a mouse model and human tissue. By utilizing Pkd1-knockout mice and AZ505, a specific inhibitor of SMYD2, they demonstrated that SMYD2 is a critical mediator of renal cyst growth. Moreover, SMYD2 induced cystic renal epithelial cell proliferation while survival was found to be mediated by STAT3 and the p65 subunit of NF- $\kappa \mathrm{B}$; activation of these two pathways is also required for the expression and activation of SMYD2 (Li et al, 2017) (Table 3). These data suggest two positive feedback loops, SMYD2/STAT3/ SMYD2 and SMYD2/NF- $\mathrm{B} / \mathrm{SMYD} 2$ in cyst development. Currently, the role of SMYD2 in other kidney diseases remains to be reported. While it has been shown that SMYD2 is also highly expressed in human kidney tumors (Pires-Luis et al., 2015), there is no report about SMYD2 expression in other human kidney diseases so far.

\section{Other Histone Methyltransferases Associated With Kidney Diseases}

Other methyltransferases have also been reported to be involved in the development of kidney diseases and pathological conditions. Wang et al. (2019) showed that overexpression of EZH1 inhibited injury of cultured renal tubular cells (HK2) in response to aristolochic acid. Fujino and Hasebe (2016) demonstrated that histone H3K4 me3 levels were elevated in the podocytes of kidneys in patients with membranous nephropathy (Table 3) and mice treated with lipopolysaccharide (LPS); treatment with siRNA specific to MLL3 (an H3K4 methyltransferase) ameliorated podocyte swelling, reduced proteinuria and improved renal function in mice treated with LPS. This suggests that MLL3 expression and H3K4 methylation may be involved in LPS-induced podocyte injury and proteinuria. Whether MLL3 also plays a role in the pathogenesis of human membranous nephropathy needs further investigation.

Recently, sequencing of whole exomes from patients with sporadic or familial focal and segmental glomerulosclerosis (FSGS) identified three new genes (SCAF1, SETD2, and LY9) that are located closer to known FSGS genes. Among them, SETD2 is a histone $\mathrm{H} 3$ lysine 36 methyltransferase, suggesting that SETD2 may contribute to FSGS. Additional studies are required for elucidating the role of SETD2 in this disease.

\section{Representative Histone Methyltransferase Inhibitors}

Given the fact that many HMTs are implicated in the pathogenesis of kidney diseases, targeting HMTs by small-molecule modulators could be effective therapy for treating them. In the past decade, many small molecules that target histone methyltransferases have been developed and used for treatment of tumors in animal models, and some of them have advanced to clinical trials. Similarly, several the histone methyltransferases inhibitors have been tested for their efficacy in the treatment of experimental kidney diseases (Table 2). Here, we summarize and highlight those histone methyltransferase inhibitors widely used in animal models of kidney diseases.

\section{EZH2 Inhibitors}

Among the EZH2 inhibitors, 3-DZNeP is frequently used in animal studies of various diseases. It can induce degradation of EZH2 and subsequently inhibits its activity. Our studies show that treatment with 3-DZNeP dose-dependently inhibited activation and proliferation of renal interstitial fibroblasts in vitro. Administration of $3-\mathrm{DZNeP}$ at as low as $2 \mathrm{mg} / \mathrm{kg}$ can effectively attenuate renal fibrosis, inhibit inflammation and lessen acute kidney injury with improvement of renal function. However, DZNeP is not a direct EZH2 inhibitor and exerts its inhibitory action through blocking S-adenosyl-L-homocysteine hydrolase. Thus, its application may lead to unwanted outcomes in SAMdependent reactions. Moreover, the lack of specificity and short half-life also limit its translation to clinical applications.

Recently, Some potent and more selective EZH2 inhibitors have been developed. Those inhibitors include GSK926, GSK343, EPZ-6438, EPZ-005687, EPZ-011989, EI1, UNC-1999, and CPI-169. All are competitive inhibitors of EZH2. Among them, GSK126 is the most potent inhibitor of EZH2. That can effectively inhibited activation of cultured renal interstitial fibroblasts (Zhou et al., 2016). In addition to the competitive inhibition of EZH2, a novel strategy to suppress EZH2 by protein degradation has been developed. For example, GNA022 can covalently bind to cYS 668 at the set domain of EZH2 and induce EZH2 degradation, thereby inhibiting tumor growth (Wang et al., 2017).

It is important to note that EPZ-6438, GSK126, and CPI1205 have already entered clinical trials (Trial number: NCT01897571, NCT02601950, NCT02601937, NCT02395601, and NCT02082977). It was found that they are well tolerated and have antitumor activity in patients with refractory B-cell non-Hodgkin lymphoma and advanced solid tumors (Yan et al., 2016). Despite great progress in clinical trials in determining the potential of EZH2 inhibitors as cancer therapy, the efficacy of those inhibitors in animal models of kidney diseases and in human kidney diseases hasn't yet been shown.

\section{G9a Inhibitors}

A number of small molecule inhibitors have been developed with the capacity to inhibit G9a catalytic activity and have been used in various in vitro and in vivo experiments. BIX01294 (diazepin-quinazolin-amine derivative), one of the first molecules 
developed to target G9a, is a competitive inhibitor specific for G9a that can reduce G9a-mediated H3K9 di-methylation, but not mono-methylation (Kubicek et al., 2007). Unlike many other HMT inhibitors, BIX-01294 competes with G9a substrate and not with G9a cofactor $S$-adenosyl-methionine (SAM), the source of the transferred methyl group. Since BIX-01294 has an intrinsic toxic effect, researchers have developed a second G9a inhibitor, UNC0638, which has high potency and specificity for G9a with lower cell toxicity. In addition, UNC0638 exhibited higher lipophilic characteristics and cell membrane permeability. Both BIX01294 and UNC0638 have been reported to inhibit cellular proliferation in various cancer cell lines, such as breast, squamous head and neck carcinoma (Artal-Martinez de Narvajas et al., 2013; Savickiene et al., 2014; Ueda et al., 2014). However, pharmacokinetics of UNC0638 is poor. Recently, Liu et al. (2013) have developed a new compound, UNC0642 that is suitable for animal studies. UNC0642 has better pharmacokinetics and maintains a high selectivity and low cell toxicity. As described in the previous section, inhibition of G9a with BIX01294 has been reported to attenuate renal fibrosis and peritoneal fibrosis in animal models. BIX01294-mediated inhibition of renal fibrosis is associated with increased expression of klotho, a renoprotective inhibitor (Irifuku et al., 2016), but BIX01294 treatment did not alter expression of klotho in renal epithelial cells (Maeda et al., 2017). The efficacy of UNC0638 and UNC0642 has not yet been evaluated in renal diseases.

\section{SUV39H1 Inhibitors}

Like methyltransferases G9a, SUV39H1 is also a H3K9 enzyme. G9a catalyzes $\mathrm{H} 3 \mathrm{~K} 9 \mathrm{me} 0$ to me1 and me1 to me2 (Tachibana et al., 2002) while SUV39H1 catalyzes di- to trimethylation of H3K9 (Lachner et al., 2001). Tri-methylated H3K9 generates a binding site for the HP1 protein, which leads to inhibition of gene transcription. Currently, chaetocin, a fungal mycotoxin originally isolated from Chaetomium minutum, has been reported to be a specific inhibitor of SUV39H1 (Greiner et al., 2005). However, subsequent studies showed that at higher concentration of chaetocin also inhibits G9a activity, suggesting it may be a nonspecific inhibitor of HMTs (Cherblanc et al., 2013). This compound has been used in both in vitro and in vivo studies It exhibits a potent cytotoxic effect in acute myeloid leukemia cells (Lai et al., 2015) and a protective effect on the heart in a murine model of cardiac injury following myocardial infarction (Yang et al., 2017a) and brain injury following cerebral ischemia (Schweizer et al., 2015). In contrast to those findings, overexpression of SUV39H1 in the kidney of $\mathrm{db} / \mathrm{db}$ models reversed the diabetic phenotype (Villeneuve et al., 2008), and administration of chaetocin increased fibronectin and p21(WAF1) protein levels in cultured mouse mesangial cells exposed to high glucose at the concentration that reduced histone H3K9me3 levels (Lin et al., 2016). As such, it appears that SUV39H1 inhibition potentiates renal injury, at least in the mouse model of DN. To date, there is still no data on the possible involvement of SUV39H1 in other animal models of kidney disease.

\section{SET7/9 Inhibitors}

Sinefungin is a small molecule inhibitor of SET7/9 that acts by competing with S-adenosyl-L-methionine and can ameliorate renal fibrosis (Sasaki et al., 2016) and peritoneal fibrosis (Tamura et al., 2018) in animal models. However, sinefungin treatment significantly reduced expression of $\mathrm{H} 3 \mathrm{~K} 4 \mathrm{me} 1$, but did not alter expression of $\mathrm{H} 3 \mathrm{~K} 4 \mathrm{me} 2$ and $\mathrm{H} 3 \mathrm{~K} 4 \mathrm{me} 3$ in the kidneys of UUO mice. In addition to sinefungin, cyproheptadine, a clinically approved antiallergy drug, has recently been identified as a Set7/9 inhibitor using a fluorogenic substrate-based HMT assay. This compound can bind Set7/9 and inhibit its enzymatic activity (Takemoto, et al., 2016). The efficacy of cyproheptadine in inhibiting tissue fibrosis, including renal fibrosis remains to be determined.

\section{DOT1I Inhibitors}

EPZ004777, EPZ5676, and SGC0946 have been reported to inhibit DOT1L. All act as competitive inhibitors of SAM, the cofactor required for the methyltransferase activity of DOT1L. EPZ004777 was first developed by Epizyme Inc. as an inhibitor of DOT1L. It shows a remarkable selectivity against other histone methyltransferases and selectively kills MLL-rearranged leukemia cells in culture (Daigle et al., 2011). Its poor pharmacokinetic properties, however, made this compound unsuitable for animal study and clinical development. A second generation DOT1L inhibitor, EPZ5676, has improved pharmacokinetic properties (Daigle et al., 2013) and has been used in animal studies and clinical trials. A first phase I study of EPZ5676 with relapsed/refractory acute leukemia has been completed; drug administration is well tolerated, and only about $15 \%$ of treated patients display adverse events. As indicated in the previous section, we also found that EPZ5676 protects against renal fibrosis. But EPZ-5676 shows low oral bioavailability and needs continuous infusion or subcutaneous injection. Both infusion and subcutaneous injection are well tolerated in animals with no overt signs of toxicity (Daigle et al., 2013). Metabolic studies showed that EPZ5676 is primarily excreted in feces and has low renal excretion (Waters et al., 2016), making EPZ5676 a suitable agent for being developed as a drug to treat kidney disease.

\section{SMYD2 Inhibitors}

LLy-507 has been reported as a potent cell-permeable and selective small molecule inhibitor of SMYD2. It has 100fold selectivity for SMYD2 over 24 other protein or DNA methyltransferases (Nguyen et al., 2015). Reports have shown that LLy-507 was effective in inhibiting proliferation of cancer cell lines in esophageal, liver, and breast cancers (Tracy et al., 2018). In the kidney, AZ505 has been reported to be effective in inhibiting cyst growth in a murine model of autosomal dominant polycystic kidney disease (Li et al., 2017).

\section{Conclusion and Future Perspectives}

Epigenetic regulation plays a vital role in the modulation of gene expression under normal and pathological conditions. Emerging evidence has shown increased expression of some HMTs such as 
EZH2, G9a, SUV39H1, and SET7/9 in several kidney diseases. Pharmacological targeting of those histone methyltransferases has been shown to attenuate renal fibrosis in animal models. Some of these molecules are also effective in interfering with the progression of diabetic nephropathy. This indicates that targeting histone methylation could be a promising therapeutic strategy for the treatment of kidney diseases. However, multiple histone methyltransferases are activated and involved in a given pathological process, and a crosstalk exists between different methylated sites, strongly suggesting that targeting one histone methyl residue or a related enzyme may lead to unpredictable effects. For example, a majority of KMTs contain a SET domain, and most inhibitors have been developed to target this domain, pointing to a potential lack of specificity leading to unwanted effects. Therefore, it is necessary to conduct further mechanistic investigations to complete the map of histone methylation in a given renal disease and develop inhibitors with higher specify and satisfactory pharmacokinetics. Moreover, since current preclinical studies related to HMT inhibitor are only conducted in limited animal models of renal disease, more research is needed to evaluate the efficacy and side effects of HMT inhibitors in different models of renal diseases and compare their efficacy and effects with those in other organs. Finally, although clinical trials

\section{REFERENCES}

Artal-Martinez de Narvajas, A., Gomez, T. S., Zhang, J. S., Mann, A. O., Taoda, Y., Gorman, J. A., et al. (2013). Epigenetic regulation of autophagy by the methyltransferase G9a. Mol. Cell Biol. 33 (20), 3983-3993. doi: 10.1128/ MCB.00813-13

Bedford, M. T., and Richard, S. (2005). Arginine methylation: an emerging regulator of protein function. Mol. Cell 18 (3), 263-272. doi: 10.1016/j. molcel.2005.04.003

Blanc, R. S., and Richard, S. (2017). Arginine methylation: the coming of age. Mol. Cell 65 (1), 8-24. doi: 10.1016/j.molcel.2016.11.003

Branscombe, T. L., Frankel, A., Lee, J. H., Cook, J. R., Yang, Z., Pestka, S., et al. (2001). PRMT5 (Janus kinase-binding protein 1) catalyzes the formation of symmetric dimethylarginine residues in proteins. J. Biol. Chem. 276 (35), 32971-32976. doi: 10.1074/jbc.M105412200

Cai, M., Bompada, P., Atac, D., Laakso, M., Groop, L., and De Marinis, Y. (2016). Epigenetic regulation of glucose-stimulated osteopontin (OPN) expression in diabetic kidney. Biochem. Biophys. Res. Commun. 469 (1), 108-113. doi: 10.1016/j.bbrc.2015.11.079

Cao, Y. P., Sun, J. Y., Li, M. Q., Dong, Y., Zhang, Y. H., Yan, J., et al. (2019). Inhibition of G9a by a small molecule inhibitor, UNC0642, induces apoptosis of human bladder cancer cells. Acta Pharmacol. Sin. 40 (8), 1076-1084. doi: 10.1038/s41401-018-0205-5

Caretti, G., Di Padova, M., Micales, B., Lyons, G. E., Sartorelli, V., et al. (2004). The Polycomb EZH2 methyltransferase regulates muscle gene expression and skeletal muscle differentiation. Genes Dev. 18 (21), 2627-2638. doi: 10.1101/ gad. 1241904

Cattaneo, P., Kunderfranco, P., Greco, C., Guffanti, A., Stirparo, G. G., and Rusconi, F. (2016). DOT1L-mediated H3K79me2 modification critically regulates gene expression during cardiomyocyte differentiation. Cell Death Differ. 23 (4), 555-564. doi: 10.1038/cdd.2014.199

Chen, D., Ma, H., Hong, H., Koh, S. S., Huang, S. M., Schurter, B. T., et al. (1999). Regulation of transcription by a protein methyltransferase. Science 284 (5423), 2174-2177.

Chen, J., Guo, Y., Zeng, W., Huang, L., Pang, Q., Nie, L., et al. (2014a). ER stress triggers MCP-1 expression through SET7/9-induced histone methylation in the kidneys of db/db mice. Am. J. Physiol. Renal Physiol. 306 (8), F916-F925. doi: 10.1152/ajprenal.00697.2012 of some EZH2 and DOT1L inhibitors for treatment of tumors have been reported (Mohammad et al., 2019), there are still no clinical trials for HMT inhibitors in renal diseases. With more evidence showing the efficacy of HMT inhibitors in animal models of kidney diseases, clinical trials with selected HMT inhibitors in renal disease are worthy of exploration.

\section{AUTHOR CONTRIBUTIONS}

CY drafted the manuscript and SZ edited it. Both authors approved it for publication.

\section{ACKNOWLEDGMENTS}

We appreciate Dr. George Bayliss for critically reading and revising this manuscript. This work was supported by the US National Institutes of Health (5R01DK085065 and 1R01DK113256-01A1 to SZ), the National Nature Science Foundation of China grants (81470920, 81830021 and 81670623 to SZ) and the Branch grant of National key grants of Ministry of Science and Technology (2018YFA0108802 to SZ).

Chen, R., Kang, R., Fan, X. G., and Tang, D. (2014b). Release and activity of histone in diseases. Cell Death Dis. 5 (8), e1370. doi: 10.1038/cddis.2014.337

Chen, Y., Yang, S., Hu, J., Yu, C., He, M., and Cai, Z. (2016). Increased expression of SETD7 promotes cell proliferation by regulating cell cycle and indicates poor prognosis in hepatocellular carcinoma. PloS One 11 (5), e0154939. doi: 10.1371/journal.pone.0154939

Chen, W. L., Sun, H. P., Li, D. D., Wang, Z. H., You, Q. D., and Guo, X. K. (2017). G9a - an appealing antineoplastic target. Curr. Cancer Drug Targets 17 (6), 555-568. doi: 10.2174/1568009616666160512145303

Cherblanc, F. L., Chapman, K. L., Reid, J., Borg, A. J., Sundriyal, S., and AlcazarFuoli, L. (2013). On the histone lysine methyltransferase activity of fungal metabolite chaetocin. J. Med. Chem. 56 (21), 8616-8625. doi: 10.1021/ jm401063r

Coward, W. R., Brand, O. J., Pasini, A., Jenkins, G., Knox, A. J., Pang, L., et al. (2018). Interplay between EZH2 and G9a regulates CXCL10 gene repression in idiopathic pulmonary fibrosis. Am. J. Respir. Cell Mol. Biol. 58 (4), 449-460. doi: 10.1165/rcmb.2017-0286OC

Daigle, S. R., Olhava, E. J., Therkelsen, C. A., Majer, C. R., Sneeringer, C. J., and Song, J., et al. (2011). Selective killing of mixed lineage leukemia cells by a potent small-molecule DOT1L inhibitor. Cancer Cell 20 (1), 53-65. doi: 10.1016/j.ccr.2011.06.009

Daigle, S. R., Olhava, E. J., Therkelsen, C. A., Basavapathruni, A., Jin, L., BoriackSjodin, P. A., et al. (2013). Potent inhibition of DOT1L as treatment of MLLfusion leukemia. Blood 122 (6), 1017-1025. doi: 10.1182/blood-2013-04-497644

Dillon, S. C., Zhang, X., Trievel, R. C., and Cheng, X. (2005). The SET-domain protein superfamily: protein lysine methyltransferases. Genome Biol. 6 (8), 227. doi: $10.1186 / \mathrm{gb}-2005-6-8-227$

Dong, C., Wu, Y., Yao, J., Wang, Y., Yu, Y., Rychahou, P. G., et al. (2012). G9a interacts with Snail and is critical for Snail-mediated E-cadherin repression in human breast cancer. J. Clin. Invest. 122 (4), 1469-1486. doi: 10.1172/JCI57349

Dressler, G. R., and Patel, S. R. (2015). Epigenetics in kidney development and renal disease. Transl. Res. 165 (1), 166-176. doi: 10.1016/j.trsl.2014.04.007

Duan, B., Bai, J., Qiu, J., Wang, J., Tong, C., Wang, X., et al. (2018). Histone-lysine $\mathrm{N}$-methyltransferase SETD7 is a potential serum biomarker for colorectal cancer patients. EBioMedicine 37, 134-143. doi: 10.1016/j.ebiom.2018.10.036

Feng, Q., Wang, H., Ng, H. H., Erdjument-Bromage, H., Tempst, P., Struhl, K., et al. (2002). Methylation of H3-lysine 79 is mediated by a new family of HMTases without a SET domain. Curr. Biol. 12 (12), 1052-1058. 
Fujino, T., and Hasebe, N. (2016). Alteration of histone H3K4 methylation in glomerular podocytes associated with proteinuria in patients with membranous nephropathy. BMC Nephrol. 17 (1), 179. doi: 10.1186/s12882-016-0390-8

Goru, S. K., Kadakol, A., Pandey, A., Malek, V., Sharma, N., and Gaikwad, A. B. (2016). Histone H2AK119 and H2BK120 mono-ubiquitination modulate SET7/9 and SUV39H1 in type 1 diabetes-induced renal fibrosis. Biochem. J. 473 (21), 3937-3949. doi: 10.1042/BCJ20160595

Grande, M. T., Sanchez-Laorden, B., Lopez-Blau, C., De Frutos, C. A., Boutet, A., Arevalo, M., et al. (2015). Snaill-induced partial epithelial-to-mesenchymal transition drives renal fibrosis in mice and can be targeted to reverse established disease. Nat. Med. 21 (9), 989-997. doi: 10.1038/nm.3901

Greer, E. L., and Shi, Y. (2012). Histone methylation: a dynamic mark in health, disease and inheritance. Nat. Rev. Genet. 13 (5), 343-357. doi: 10.1038/nrg3173

Greiner, D., Bonaldi, T., Eskeland, R., Roemer, E., and Imhof, A. (2005). Identification of a specific inhibitor of the histone methyltransferase SU(VAR)3-9. Nat. Chem. Biol. 1 (3), 143-145. doi: 10.1038/nchembio721

Guo, Q., Li, X., Han, H., Li, C., Liu, S., Gao, W., et al. (2016). Histone lysine methylation in TGF-beta1 mediated $\mathrm{p} 21$ gene expression in rat mesangial cells. BioMed. Res. Int. 2016, 6927234. doi: 10.1155/2016/6927234

Herz, H. M., Garruss, A., and Shilatifard, A. (2013). SET for life: biochemical activities and biological functions of SET domain-containing proteins. Trends Biochem. Sci. 38 (12), 621-639. doi: 10.1016/j.tibs.2013.09.004

Hua, K. T., Wang, M. Y., Chen, M. W., Wei, L. H., Chen, C. K., Ko, C. H., et al. (2014). The H3K9 methyltransferase G9a is a marker of aggressive ovarian cancer that promotes peritoneal metastasis. Mol. Cancer 13, 189. doi: 10.1186/1476-4598-13-189

Huang, J., Dorsey, J., Chuikov, S., Perez-Burgos, L., Zhang, X., Jenuwein, T., et al. (2010). G9a and Glp methylate lysine 373 in the tumor suppressor p53. J. Biol. Chem. 285 (13), 9636-9641. doi: 10.1074/jbc.M109.062588

Huang, R., Li, X., Yu, Y., Ma, L., Liu, S., Zong, X., et al. (2017). SETD7 is a prognosis predicting factor of breast cancer and regulates redox homeostasis. Oncotarget 8 (55), 94080-94090. doi: 10.18632/oncotarget.21583

Huyen, Y., Zgheib, O., Ditullio, R. A. Jr., Gorgoulis, V. G., Zacharatos, P., and Petty, T. J. (2004). Methylated lysine 79 of histone H3 targets 53BP1 to DNA doublestrand breaks. Nature 432 (7015), 406-411. doi: 10.1038/nature03114

Irifuku, T., Doi, S., Sasaki, K., Doi, T., Nakashima, A., and Ueno, T. (2016). Inhibition of H3K9 histone methyltransferase G9a attenuates renal fibrosis and retains klotho expression. Kidney Int. 89 (1), 147-157. doi: 10.1038/ki.2015.291

Janzen, C. J., Hake, S. B., Lowell, J. E., and Cross, G. A. (2006). Selective di- or trimethylation of histone $\mathrm{H} 3$ lysine 76 by two DOT1 homologs is important for cell cycle regulation in Trypanosoma brucei. Mol. Cell 23 (4), 497-507. doi: 10.1016/j.molcel.2006.06.027

Jones, B., Su, H., Bhat, A., Lei, H., Bajko, J., Hevi, S., et al. (2008). The histone H3K79 methyltransferase Dot1L is essential for mammalian development and heterochromatin structure. PloS Genet. 4 (9), e1000190. doi: 10.1371/journal. pgen. 1000190

Kubicek, S., O'Sullivan, R. J., August, E. M., Hickey, E. R., Zhang, Q., Teodoro, M. L., et al. (2007). Reversal of H3K9me2 by a small-molecule inhibitor for the G9a histone methyltransferase. Mol. Cell 25 (3), 473-481. doi: 10.1016/j.molcel.2007.01.017

Kurash, J. K., Lei, H., Shen, Q., Marston, W. L., Granda, B. W., Fan, H., et al. (2008). Methylation of $\mathrm{p} 53$ by Set7/9 mediates $\mathrm{p} 53$ acetylation and activity in vivo. Mol. Cell 29 (3), 392-400. doi: 10.1016/j.molcel.2007.12.025

Lachner, M., O'Carroll, D., Rea, S., Mechtler, K., and Jenuwein, T. (2001). Methylation of histone H3 lysine 9 creates a binding site for HP1 proteins. Nature 410 (6824), 116-120. doi: 10.1038/35065132

Lai, Y. S., Chen, J. Y., Tsai, H. J., Chen, T. Y., and Hung, W. C. (2015).The SUV39H1 inhibitor chaetocin induces differentiation and shows synergistic cytotoxicity with other epigenetic drugs in acute myeloid leukemia cells. Blood Cancer J. 5, e313. doi: 10.1038/bcj.2015.37

Li, B., Carey, M., and Workman, J. L. (2007). The role of chromatin during transcription. Cell 128 (4), 707-719. doi: 10.1016/j.cell.2007.01.015

Li, Y., Reddy, M. A., Miao, F., Shanmugam, N., Yee, J. K., Hawkins, D., et al. (2008). Role of the histone $\mathrm{H} 3$ lysine 4 methyltransferase, SET7/9, in the regulation of NF-kappaB-dependent inflammatory genes. Relevance to diabetes and inflammation. J. Biol. Chem. 283 (39), 26771-26781. doi: 10.1074/jbc.M802800200

Li, L., Zhang, Y., Xu, M., Rong, R., Wang, J., and Zhu, T. (2016a). Inhibition of histone methyltransferase EZH2 ameliorates early acute renal allograft rejection in rats. BMC Immunol. 17 (1), 41. doi: 10.1186/s12865-016-0179-3
Li, M. F., Zhang, R., Li, T. T., Chen, M. Y., Li, L. X., Lu, J. X., et al. (2016b). High Glucose increases the expression of inflammatory cytokine genes in macrophages through $\mathrm{H} 3 \mathrm{~K} 9$ methyltransferase mechanism. J. Interferon Cytokine Res. 36 (1), 48-61. doi: 10.1089/jir.2014.0172

Li, L. X., Fan, L. X., Zhou, J. X., Grantham, J. J., Calvet, J. P., Sage, J., et al. (2017). Lysine methyltransferase SMYD2 promotes cyst growth in autosomal dominant polycystic kidney disease. J. Clin. Invest. 127 (7), 2751-2764. doi: 10.1172/JCI90921

Liang, H., Huang, Q., Liao, M. J., Xu, F., Zhang, T., He, J., et al. (2019). EZH2 plays a crucial role in ischemia/reperfusion-induced acute kidney injury by regulating p38 signaling. Inflammation Res. 68 (4), 325-336. doi: 10.1007/ s00011-019-01221-3

Lin, S. H., Ho, W. T., Wang, Y. T., Chuang, C. T., Chuang, L. Y., and Guh, J. Y. (2016). Histone methyltransferase Suv39h1 attenuates high glucose-induced fibronectin and p21(WAF1) in mesangial cells. Int. J. Biochem. Cell Biol. 78, 96-105. doi: 10.1016/j.biocel.2016.06.021

Liu, F., Barsyte-Lovejoy, D., Li, F., Xiong, Y., Korboukh, V., Huang, X. P., et al. (2013). Discovery of an in vivo chemical probe of the lysine methyltransferases G9a and GLP. J. Med. Chem. 56 (21), 8931-8942. doi: 10.1021/jm401480r

Liu, L., Zou, J., Guan, Y., Zhang, Y., Zhang, W., Zhou, X., et al. (2019). Blocking the histone lysine 79 methyltransferase DOT1L alleviates renal fibrosis through inhibition of renal fibroblast activation and epithelial-mesenchymal transition. FASEB J. 33 (11), 11941-11958. doi: 10.1096/fj.201801861R

Lovisa, S., LeBleu, V. S., Tampe, B., Sugimoto, H., Vadnagara, K., Carstens, J. L., et al. (2015). Epithelial-to-mesenchymal transition induces cell cycle arrest and parenchymal damage in renal fibrosis. Nat. Med. 21 (9), 998-1009. doi: 10.1038/nm.3902

Maeda, K., Doi, S., Nakashima, A., Nagai, T., Irifuku, T., Ueno, T., et al. (2017). Inhibition of H3K9 methyltransferase G9a ameliorates methylglyoxalinduced peritoneal fibrosis. PloS One 12 (3), e0173706. doi: 10.1371/journal. pone. 0173706

Majumder, S., Thieme, K., Batchu, S. N., Alghamdi, T. A., Bowskill, B. B., Kabir, M. G., et al. (2018). Shifts in podocyte histone H3K27me3 regulate mouse and human glomerular disease. J. Clin. Invest. 128 (1), 483-499. doi: 10.1172/JCI95946

Martinez, A. M., and Cavalli, G. (2006). The role of polycomb group proteins in cell cycle regulation during development. Cell Cycle 5 (11), 1189-1197. doi: $10.4161 /$ cc.5.11.2781

McBride, A. E., Weiss, V. H., Kim, H. K., Hogle, J. M., and Silver, P. A. (2000). Analysis of the yeast arginine methyltransferase Hmt1p/Rmtlp and its in vivo function. Cofactor binding and substrate interactions. J. Biol. Chem. 275 (5), 3128-3136.

McGrath, J., and Trojer, P. (2015). Targeting histone lysine methylation in cancer. Pharmacol. Ther. 150, 1-22. doi: 10.1016/j.pharmthera.2015.01.002

McLaughlin, N., Wang, F., Saifudeen, Z., and El-Dahr, S. S. (2014). In situ histone landscape of nephrogenesis. Epigenetics 9 (2), 222-235. doi: 10.4161/epi.26793

Min, J., Feng, Q., Li, Z., Zhang, Y., and Xu, R. M. (2003). Structure of the catalytic domain of human DOT1L, a non-SET domain nucleosomal histone methyltransferase. Cell 112 (5), 711-723.

Mohammad, H. P., Barbash., O., and Creasy, C. L. (2019). Targeting epigenetic modifications in cancer therapy: erasing the roadmap to cancer. Nat. Med. 25 (3), 403-418. doi: 10.1038/s41591-019-0376-8

Ng, H. H., Feng, Q., Wang, H., Erdjument-Bromage, H., Tempst, P., Zhang, Y., et al. (2002). Lysine methylation within the globular domain of histone H3 by Dot1 is important for telomeric silencing and Sir protein association. Genes Dev. 16 (12), 1518-1527. doi: 10.1101/gad.1001502

Nguyen, H., Allali-Hassani, A., Antonysamy, S., Chang, S., Chen, L. H., Curtis, C., et al. (2015). LLY-507, a Cell-active, Potent, and Selective Inhibitor of Proteinlysine Methyltransferase SMYD2. J. Biol. Chem. 290 (22), 13641-13653. doi: 10.1074/jbc.M114.626861

Ni, J., Hou, X., Wang, X., Shi, Y., Xu, L., Zheng, X. , et al. (2019). 3-deazaneplanocin A protects against cisplatin-induced renal tubular cell apoptosis and acute kidney injury by restoration of E-cadherin expression. Cell Death Dis. 10 (5), 355. doi: 10.1038/s41419-019-1589-y

Ningxia, Z., Shuyan, L., Zhongjing, S., Ling, C., Tianzhong, M., Lifeng, W., et al. (2011). The expression pattern of polycomb group protein Ezh2 during mouse embryogenesis. Anat. Rec. (Hoboken) 294 (7), 1150-1157. doi: 10.1002/ar.21416

Oktaba, K., Gutierrez, L., Gagneur, J., Girardot, C., Sengupta, A. K., Furlong, E. E., et al. (2008). Dynamic regulation by polycomb group protein complexes controls 
pattern formation and the cell cycle in Drosophila. Dev. Cell 15 (6), 877-889. doi: 10.1016/j.devcel.2008.10.005

Peschansky, V. J., and Wahlestedt, C. (2014). Non-coding RNAs as direct and indirect modulators of epigenetic regulation. Epigenetics 9 (1), 3-12. doi: 10.4161/Epi.27473

Pires-Luis, A. S., Vieira-Coimbra, M., Vieira, F. Q., Costa-Pinheiro, P., SilvaSantos, R., Dias, P. C., et al. (2015). Expression of histone methyltransferases as novel biomarkers for renal cell tumor diagnosis and prognostication. Epigenetics 10 (11), 1033-1043. doi: 10.1080/15592294.2015.1103578

Portela, A., and Esteller, M. (2010). Epigenetic modifications and human disease. Nat. Biotechnol. 28 (10), 1057-1068. doi: 10.1038/nbt.1685

Rea, S., Eisenhaber, F., O'Carroll, D., Strahl, B. D., Sun, Z. W., Schmid, M., et al. (2000). Regulation of chromatin structure by site-specific histone H3 methyltransferases. Nature 406 (6796), 593-599. doi: 10.1038/35020506

Reddy, M. A., and Natarajan, R. (2015). Recent developments in epigenetics of acute and chronic kidney diseases. Kidney Int. 88 (2), 250-261. doi: 10.1038/ ki.2015.148

Sasaki, K., Doi, S., Nakashima, A., Irifuku, T., Yamada, K., Kokoroishi, K., et al. (2016). Inhibition of SET Domain-Containing Lysine Methyltransferase 7/9 Ameliorates Renal Fibrosis. J. Am. Soc. Nephrol. 27 (1), 203-215. doi: 10.1681/ ASN.2014090850

Savickiene, J., Treigyte, G., Stirblyte, I., Valiuliene, G., and Navakauskiene, R. (2014). Euchromatic histone methyltransferase 2 inhibitor, BIX-01294, sensitizes human promyelocytic leukemia HL-60 and NB4 cells to growth inhibition and differentiation. Leuk Res. 38 (7), 822-829. doi: 10.1016/j. leukres.2014.04.003

Schweizer, S., Harms, C., Lerch, H., Flynn, J., Hecht, J., Yildirim, F., et al. (2015). Inhibition of histone methyltransferases SUV39H1 and G9a leads to neuroprotection in an in vitro model of cerebral ischemia. J. Cereb. Blood Flow Metab. 35 (10), 1640-1647. doi: 10.1038/jcbfm.2015.99

Shi, Y., Xu, L., Tao, M., Fang, L., Lu, J., Gu, H., et al. (2019). Blockade of enhancer of zeste homolog 2 alleviates renal injury associated with hyperuricemia. Am. J. Physiol. Renal Physiol. 316 (3), F488-F505. doi: 10.1152/ajprenal.00234.2018

Shuttleworth, V. G., Gaughan, L., Nawafa, L., Mooney, C. A., Cobb, S. L., Sheerin, N. S., et al. (2018). The methyltransferase SET9 regulates TGFB1 activation of renal fibroblasts via interaction with SMAD3. J. Cell Sci. 131 (1), jcs207761. doi: $10.1242 /$ jcs. 207761

Siddiqi, F. S., Majumder, S., Thai, K., Abdalla, M., Hu, P., Advani, S. L., et al. (2016). The histone methyltransferase enzyme enhancer of zeste homolog 2 protects against podocyte oxidative stress and renal injury in diabetes. J. Am. Soc. Nephrol. 27 (7), 2021-2034. doi: 10.1681/ASN.2014090898

Sims, R. J., Nishioka, K., and Reinberg, D. (2003). Histone lysine methylation: a signature for chromatin function. Trends Genet. 19 (11), 629-639. doi: 10.1016/j.tig.2003.09.007

Sun, G., Reddy, M. A., Yuan, H., Lanting, L., Kato, M., and Natarajan, R. (2010). Epigenetic histone methylation modulates fibrotic gene expression. J. Am. Soc. Nephrol. 21 (12), 2069-2080. doi: 10.1681/ASN.2010060633

Susztak, K. (2014). Understanding the epigenetic syntax for the genetic alphabet in the kidney. J. Am. Soc. Nephrol. 25 (1), 10-17. doi: 10.1681/ASN.2013050461

Tachibana, M., Sugimoto, K., Fukushima, T., and Shinkai, Y. (2001). Set domaincontaining protein, G9a, is a novel lysine-preferring mammalian histone methyltransferase with hyperactivity and specific selectivity to lysines 9 and 27 of histone H3. J. Biol. Chem. 276 (27), 25309-25317. doi: 10.1074/jbc. M101914200

Tachibana, M., Sugimoto, K., Nozaki, M., Ueda, J., Ohta, T., Ohki, M., et al. (2002). G9a histone methyltransferase plays a dominant role in euchromatic histone H3 lysine 9 methylation and is essential for early embryogenesis. Genes Dev. 16 (14), 1779-1791. doi: 10.1101/gad.989402

Takawa, M., Masuda, K., Kunizaki, M., Daigo, Y., Takagi, K., Iwai, Y., et al. (2011). Validation of the histone methyltransferase EZH2 as a therapeutic target for various types of human cancer and as a prognostic marker. Cancer Sci. 102 (7), 1298-1305. doi: 10.1111/j.1349-7006.2011.01958.x

Takemoto, Y., Ito, A., Niwa, H., Okamura, M., Fujiwara, T., Hirano, T., et al. (2016). Identification of Cyproheptadine as an Inhibitor of SET Domain Containing Lysine Methyltransferase 7/9 (Set7/9) That Regulates EstrogenDependent Transcription. J. Med. Chem. 59 (8), 3650-3660. doi: 10.1021/acs. jmedchem.5b01732
Tamura, R., Doi, S., Nakashima, A., Sasaki, K., Maeda, K., Ueno, T., et al. (2018). Inhibition of the H3K4 methyltransferase SET7/9 ameliorates peritoneal fibrosis. PloS One 13 (5), e0196844. doi: 10.1371/journal.pone.0196844

Tracy, C., Warren, J. S., Szulik, M., Wang, L., Garcia, J., Makaju, A., et al. (2018). The SMYD family of methyltransferases: role in cardiac and skeletal muscle physiology and pathology. Curr. Opin. Physiol. 1, 140-152. doi: 10.1016/j.cophys.2017.10.001

Ueda, J., Ho, J. C., Lee, K. L., Kitajima, S., Yang, H., Sun, W., et al. (2014). The hypoxia-inducible epigenetic regulators Jmjdla and G9a provide a mechanistic link between angiogenesis and tumor growth. Mol. Cell Biol. 34 (19), 37023720. doi: 10.1128/MCB.00099-14

Vignais, M. L., Sadowski, H. B., Watling, D., Rogers, N. C., and Gilman, M. (1996). Platelet-derived growth factor induces phosphorylation of multiple JAK family kinases and STAT proteins. Mol. Cell Biol. 16 (4), 1759-1769.

Villeneuve, L. M., Reddy, M. A., Lanting, L. L., Wang, M., Meng, L., and Natarajan, R. (2008). Epigenetic histone H3 lysine 9 methylation in metabolic memory and inflammatory phenotype of vascular smooth muscle cells in diabetes. Proc. Natl. Acad. Sci. U.S.A. 105 (26), 9047-9052. doi: 10.1073/pnas.0803623105

Wakeman, T. P., Wang, Q., Feng, J., and Wang, X. F. (2012). Bat3 facilitates H3K79 dimethylation by DOT1L and promotes DNA damage-induced 53BP1 foci at G1/G2 cell-cycle phases. EMBO J. 31 (9), 2169-2181. doi: 10.1038/emboj.2012.50

Wang, W., Koka, V., and Lan, H. Y. (2005). Transforming growth factor-beta and Smad signalling in kidney diseases. Nephrol. (Carlton) 10 (1), 48-56. doi: 10.1111/j.1440-1797.2005.00334.x

Wang, X., Cao, W., Zhang, J., Yan, M., Xu, Q., Wu, X., et al. (2017). A covalently bound inhibitor triggers EZH2 degradation through CHIP-mediated ubiquitination. EMBO J. 36 (9), 1243-1260. doi: 10.15252/embj.201694058

Wang, C., Shu, L., Zhang, C., Li, W., Wu, R., Guo, Y., et al. (2018a). Histone Methyltransferase Setd7 Regulates Nrf2 Signaling Pathway by Phenethyl Isothiocyanate and Ursolic Acid in Human Prostate Cancer Cells. Mol. Nutr. Food Res. 62 (18), e1700840. doi: 10.1002/mnfr.201700840

Wang, J., Yan, W., Peng, X., Jiang, Y., He, L., Peng, Y., et al. (2018b). Functional Role of SUV39H1 in Human Renal Tubular Epithelial Cells Under High-glucose Ambiance. Inflammation 41 (1), 1-10. doi: 10.1007/s10753-017-0657-7

Wang, L., Liu, N., Xue, X., and Zhou, S. (2019). The Effect of Overexpression of the Enhancer of Zeste Homolog 1 (EZH1) Gene on Aristolochic Acid-Induced Injury in HK-2 Human Kidney Proximal Tubule Cells In Vitro. Med. Sci. Monit. 25, 801-810. doi: 10.12659/MSM.911611

Wang, M., Chun, J., Genovese, G., Knob, A. U., Benjamin, A., Wilkins, M. S., et al. (2019). Contributions of Rare Gene Variants to Familial and Sporadic FSGS. J. Am. Soc. Nephrol. doi: 10.1681/ASN.2019020152

Waters, N. J., Smith, S. A., Olhava, E. J., Duncan, K. W., Burton, R. D., and O’Neill, J. (2016). Metabolism and disposition of the DOT1L inhibitor, pinometostat (EPZ-5676), in rat, dog and human. Cancer Chemother. Pharmacol. 77 (1), 43-62. doi: 10.1007/s00280-015-2929-y

Wen, L., Fu, L., Guo, X., Chen, Y., and Shi, Y. B. (2015). Histone methyltransferase Dot1L plays a role in postembryonic development in Xenopus tropicalis. FASEB J. 29 (2), 385-393. doi: 10.1096/fj.14-252171

Wong, M., Polly, P., and Liu, T. (2015). The histone methyltransferase DOT1L: regulatory functions and a cancer therapy target. Am. J. Cancer Res. 5 (9), 2823-2837.

Wood, A., and Shilatifard, A. (2004). Posttranslational modifications of histones by methylation. Adv. Protein Chem. 67, 201-222. doi: 10.1016/ S0065-3233(04)67008-2

Wu, H., Chen, L., Zhang, X., Zhou, Q., Li, J. M., Berger, S., et al. (2013). Aqp5 is a new transcriptional target of Dotla and a regulator of Aqp2. PloS One 8 (1), e53342. doi: 10.1371/journal.pone.0053342

Wu, Y. S., Chen, Y. T., Bao, Y. T., Li, Z. M., Zhou, X. J., He, J. N., et al. (2016). Identification and verification of potential therapeutic target genes in berberine-treated zucker diabetic fatty rats through bioinformatics analysis. PloS One 11 (11), e0166378. doi: 10.1371/journal.pone.0166378

Yan, W., Herman, J. G., Guo, M., et al. (2016). Epigenome-based personalized medicine in human cancer. Epigenomics 8 (1), 119-133. doi: 10.2217/epi.15.84

Yang, G., Weng, X., Zhao, Y., Zhang, X., Hu, Y., and Dai, X. (2017a). The histone H3K9 methyltransferase SUV39H links SIRT1 repression to myocardial infarction. Nat. Commun. 8, 14941. doi: 10.1038/ncomms14941

Yang, G., Zhang, X., Weng, X., Liang, P., Dai, X., Zeng, S., et al. (2017b). SUV39H1 mediated SIRT1 trans-repression contributes to cardiac ischemia-reperfusion injury. Basic Res. Cardiol. 112 (3), 22. doi: 10.1007/ s00395-017-0608-3 
Yu, H., Lin, Q., Wang, Y., He, Y., Fu, S., Jiang, H., et al. (2013). Inhibition of H3K9 methyltransferases G9a/GLP prevents ototoxicity and ongoing hair cell death. Cell Death Dis. 4, e506. doi: 10.1038/cddis.2013.28

Zhang, J., He, P., Xi, Y., Geng, M., Chen, Y., and Ding, J. (2015). Down-regulation of G9a triggers DNA damage response and inhibits colorectal cancer cells proliferation. Oncotarget 6 (5), 2917-2927. doi: 10.18632/oncotarget.2784

Zhou, Q., Liu, K., Wu, H., Chen, L., Pouranan, V., Yuan, M. construed (2012). Spironolactone rescues Dotla-Af9-mediated repression of endothelin-1 and improves kidney injury in streptozotocin-induced diabetic rats. PloS One 7 (10), e47360. doi: 10.1371/journal.pone.0047360

Zhou, X., Zang, X., Ponnusamy, M., Masucci, M. V., Tolbert, E., and Gong, R. (2016). Enhancer of Zeste Homolog 2 Inhibition Attenuates Renal Fibrosis by Maintaining Smad7 and Phosphatase and Tensin Homolog Expression. J. Am. Soc. Nephrol. 27 (7), 2092-2108. doi: 10.1681/ASN.2015040457

Zhou, X., Xiong, C., Tolbert, E., Zhao, T. C., Bayliss, G., Zhuang, S., et al. (2018a). Targeting histone methyltransferase enhancer of zeste homolog-2 inhibits renal epithelial-mesenchymal transition and attenuates renal fibrosis. FASEB J. 32 (11), 5976-5989. doi: 10.1096/ff.201800237R

Zhou, X., Zang, X., Guan, Y., Tolbert, T., Zhao, T. C., Bayliss, G., et al. (2018b). Targeting enhancer of zeste homolog 2 protects against acute kidney injury. Cell Death Dis. 9 (11), 1067. doi: 10.1038/s41419-018-1012-0

Conflict of Interest: The authors declare that the research was conducted in the absence of any commercial or financial relationships that could be construed as a potential conflict of interest.

Copyright (c) $2019 \mathrm{Yu}$ and Zhuang. This is an open-access article distributed under the terms of the Creative Commons Attribution License (CC BY). The use, distribution or reproduction in other forums is permitted, provided the original author(s) and the copyright owner(s) are credited and that the original publication in this journal is cited, in accordance with accepted academic practice. No use, distribution or reproduction is permitted which does not comply with these terms. 